TITLE:

\title{
Changes in hydrophobic and hydrophilic hydration properties caused by raising the pressure or by lowering the temperature
}

\author{
$\operatorname{AUTHOR}(S)$ :
}

Kinoshita, Masahiro; Oshima, Hiraku

\section{CITATION:}

Kinoshita, Masahiro ... [et al]. Changes in hydrophobic and hydrophilic hydration properties caused by raising the pressure or by lowering the temperature. Chemical Physics Letters 2014, 610-611: 1-7

ISSUE DATE:

2014-08-28

URL:

http://hdl.handle.net/2433/189416

\section{RIGHT:}

(C) 2014 Elsevier B.V.; This is not the published version. Please cite only the published version.; この論文は出版社版でありません。引用の際に は出版社版をご確認ご利用ください。 


\title{
Changes in hydrophobic and hydrophilic hydration properties caused by raising the pressure or by lowering the temperature
}

\author{
Masahiro Kinoshita and Hiraku Oshima \\ Institute of Advanced Energy, Kyoto University, Uji, Kyoto 611-0011, Japan
}

Author to whom correspondence should be addressed: Masahiro Kinoshita.

E-mail address: kinoshit@iae.kyoto-u.ac.jp.

\begin{abstract}
We analyze entropic and energetic components of the hydration free energy (HFE) for hydrophobic and hydrophilic solutes under isochoric condition using the angle-dependent integral equation theory combined with a multipolar water model. The entropic component, which always makes a positive contribution to the HFE, becomes larger at high pressures and smaller at low temperatures for both solutes: It governs the pressure and temperature dependence of the HFE. For hydrophilic solutes at low temperatures, however, the energetic component becomes more important and makes them less hydrophilic. We discuss the validity of our previous studies on pressure and cold denaturating of proteins.
\end{abstract}




\section{Introduction}

A protein folds into a unique structure (i.e., the native structure) in aqueous solution under the physiological condition. However, it collapses by raising the pressure or by lowering the temperature. Investigating these processes will provide physical insights into the folding/unfolding mechanism of proteins. In earlier works, we developed physical pictures of pressure [1] and cold [2-4] denaturating of proteins.

Our physical picture of pressure denaturation can be summarized as follows [1]. We first consider a spherical solute. The presence of a solute in water generates a volume from which the centers of water molecules are excluded (Fig. 1(a)). A water molecule also generates an excluded volume for the other water molecules (Fig. 1(b)), and water molecules are thus entropically correlated (i.e., the water crowding, which originates from the water-water pair, water-water-water triplet, and higher-order correlations, comes into play). When water molecules contact the solute, the translational freedom of the water molecules in contact with the solute decreases, leading to their entropic loss. This description is based on the solute-water pair correlation [5]. However, the overlap of excluded volumes occurs as shown in Fig. 1(c) and the total volume available to the other water molecules increases, that is, their crowding reduces. The reduction brings their entropic gain. This description is based on the solute-water-water triplet and higher-order (i.e., solute-water many-body) correlation [5]. At low pressures, the water crowding is moderate and reducing it is not crucial. Consequently, the water molecules contacting the solute are not many. At high pressures, by contrast, the water crowding is serious and reducing it is crucial with the result that many water molecules are forced to contact the solute. At high pressures, when the solute possesses a flexible, polyatomic structure as in the case of a protein, its structure is changed to the one with a much larger water-accessible surface area for largely increasing the number of water molecules in contact with the protein and an excluded volume kept sufficiently small: i.e., the swelling, pressure-denatured structure. The denaturation leads to an increase in the total water entropy. Characteristics of pressure denaturation of a protein are illustrated in Fig. 2.

The physical picture of pressure denaturation described above was developed on the basis of theoretical analyses considering only the entropic component of the hydration free energy (HFE). Moreover, a protein comprising only hydrophobic groups was treated. The following questions then arise: How does the energetic component of the HFE behave?; and how is the result influenced by the presence of hydrophilic groups in a protein?

Our physical picture of cold denaturation can be summarized as follows [2-4]. We find that the principal driving force for protein folding is a water-entropy gain arising from the reduction of water crowding in the bulk. This reduction originates from the protein-water many-body correlation [5]. At low temperatures the driving force is considerably weakened, yielding to the other factors opposing to the folding. The 
protein is then denatured, which is cold denaturation. The denaturation brings a water-entropy loss, but the sum of the protein conformational-entropy gain and the decrease in the system energy dominates. The weakening of the driving force for protein folding at low temperatures is ascribed to enhanced local association of water molecules arising from the strengthened hydrogen bonding in the bulk, giving rise to more inhomogeneity followed by the formation of more void space: Water can accommodate a large solute with less difficulty. Though the denaturation is accompanied by a decrease in the water energy including the protein-water interaction energy (see Sec. 3.1) and an increase in the protein intramolecular energy, the former is larger. It is true that the water near the protein becomes more structured (i.e., the number of hydrogen bonds increases and/or the hydrogen bonding is strengthened) when the temperature is lowered. However, the entropy decrease due to the structuring at low temperatures is cancelled out by the energy lowering accompanied in terms of the free-energy change. The cold denatured state is considerably more extended than the pressure-denatured state. Characteristics of cold denaturation of a protein are illustrated in Fig. 3.

In the development of the physical picture of cold denaturation described above, both of the entropic and energetic components of the HFE were taken into account, but a protein comprising only hydrophobic groups was treated. An important concern is the effect of the presence of hydrophilic groups in a protein. The effective interaction between a hydrophilic group and a water molecule is determined primarily by the electrostatic attractive interaction scaled by $k_{\mathrm{B}} T$ ( $k_{\mathrm{B}}$ is Boltzmann's constant and $T$ is the absolute temperature). A decrease in $T$ leads to an enhanced effective attractive interaction. Therefore, one might think the following: The power of exposing hydrophilic groups to water becomes stronger by the temperature lowering, inducing (or at least facilitating) cold denaturation.

In the present Letter, to answer the questions raised above or clarify the uncertain points, we analyze entropic and energetic components of the HFE for hydrophobic and hydrophilic solutes using the angle-dependent integral equation theory combined with a multipolar water model. We consider isochoric condition in the analysis for the following reasons: It is free from the effects of compression or expansion of the bulk water and more suited to physical interpretation of a change in a thermodynamic quantity of hydration [6-8]; and it is much more convenient in a theoretical treatment. We show that the results obtained certainly support the validity of our theoretical approaches to the elucidation of pressure and cold denaturating.

We skip a review of the previously reported physical pictures of protein denaturation which are more or less different from ours because we do not treat the denaturation itself (they were reviewed in our earlier publications ${ }^{1-5}$ ). As for the hydrophobic and hydrophilic hydrations, there are a number of experimental, theoretical, and computer simulation studies reported in the literature which can hardly be reviewed in a letter. However, the present study can be distinguished from the 
others in the following senses: (1) We consider the hydrophobic and hydrophilic hydrations at elevated pressures or lowered temperatures which are relevant to pressure or cold denaturating of proteins; and (2) all of the three quantities, the hydration free energy, energy, and entropy, are calculated using a realistic water model. To the best of our knowledge, there are no studies satisfying both of these two points.

It is worthwhile to clarify our standpoint. Suppose that a solute is considered and solute-water electrostatic (attractive) potential is not incorporated in the calculation. When it is incorporated, the hydration properties exhibit drastic changes. However, we are not concerned with such changes. A principal concern is the following: How are the effects of solute-water electrostatic potential varied by raising the pressure or by lowering the temperature? In arguing the physical origin of pressure or cold denaturating of proteins, we have to account for the factors whose effects are sensitive to the pressure or temperature change. Even when a factor has large effects on solute hydration properties or changes in the thermodynamic quantities upon protein folding or unfolding, if its effects are insensitive to the pressure or temperature change, it can be neglected.

\section{Model and Theory}

\subsection{Water model}

A water molecule is modeled as a hard sphere with diameter $d_{S}=0.28 \mathrm{~nm}$ in which a point dipole and a point quadrupole of tetrahedral symmetry are embedded $[9,10]$. The influence of molecular polarizability of water is included by employing the self-consistent mean field (SCMF) theory $[9,10]$. At the SCMF level the many-body induced interactions are reduced to pairwise additive potentials involving an effective dipole moment. The effective dipole moment thus determined at $298 \mathrm{~K}$ and $1 \mathrm{~atm}$ is about 1.42 times larger than the bare gas-phase dipole moment.

To look at the pressure dependence of hydration properties of solutes, $T$ is set at $298 \mathrm{~K}$, and 0.7317 and 0.8500 are considered for the reduced number density $\rho_{\mathrm{s}} d_{\mathrm{s}}{ }^{3}$ at low and high pressures, respectively [1]. The effective dipole moment at the high pressure is only $\sim 0.8 \%$ larger than that at the low one.

Three temperatures, 258, 273, and $298 \mathrm{~K}$, are tested for examining the temperature dependence of hydration properties of solutes at low pressures [2-4]. 323 $\mathrm{K}$ is additionally tested for the hydrophilic solute considered. The number density of the solvent is taken to be that of real water along the saturation curve at $1 \mathrm{~atm}$. The value at the lowest temperature is estimated by the extrapolation. The values of $\rho_{\mathrm{s}} d_{\mathrm{s}}{ }^{3}$ ( $\rho_{\mathrm{S}}$ is the number density of water) at $258,273,298$, and $323 \mathrm{~K}$ are $0.7312,0.7338$, 0.7317 , and 0.7251 , respectively. The effective dipole moment becomes smaller with increasing $T$. 


\subsection{Models of hydrophobic and hydrophilic solutes}

The solute is modeled as a hard sphere with diameter $d_{U}$ without or with a point charge $q_{U}$ placed at its center. The subscripts, "S" and "U", respectively, represent "solvent (water)" and "solute". Detailed results are given for the two solutes: A hydrophobic solute with $d_{\mathrm{U}}=2 d_{\mathrm{S}}$ and $q_{\mathrm{U}}=0$ and a hydrophilic solute with $d_{\mathrm{U}}=2 d_{\mathrm{S}}$ and $q_{\mathrm{U}}=e$ ( $e$ is the elementary electric charge). These solutes were treated in our earlier works [11,12] and shown to exhibit typical hydrophobic and hydrophilic hydration properties, respectively. Some more different values of $d_{\mathrm{U}}$ and $q_{\mathrm{U}}$, which are similar to those of oxygen and nitrogen atoms in a protein, are also examined for hydrophilic hydration and the effects of $d_{\mathrm{U}}$ and $q_{\mathrm{U}}$ are qualitatively discussed (the detailed results are not given).

\subsection{Angle-dependent integral equation theory}

The Ornstein-Zernike (OZ) equation for the mixture comprising water molecules and spherical solutes can be written as [6,9-12]

$$
\begin{aligned}
& \eta_{\alpha \beta}(12)=\left\{1 /\left(8 \pi^{2}\right)\right\} \sum_{\gamma} \rho_{\gamma} \int_{C \alpha \gamma}(13)\left\{\eta_{\gamma \beta}(32)+c_{\gamma \beta}(32)\right\} d(3), \\
& \eta_{\alpha \beta}(12)=h_{\alpha \beta}(12)-c_{\alpha \beta}(12) ; \alpha, \beta=\mathrm{S}, \mathrm{U},
\end{aligned}
$$

where $h$ and $c$ are the total and direct correlation functions, respectively, (ij) represents ( $\boldsymbol{r}_{i j}, \boldsymbol{\Omega}_{i}, \boldsymbol{\Omega}_{j}$ ), $\boldsymbol{r}_{i j}$ is the vector connecting the centers of particles $i$ and $j, \boldsymbol{\Omega}_{i}$ denotes the three Euler angles describing the orientation of particle $i, \int d(3)$ represents integration over all position and angular coordinates of particle 3 , and $\rho$ is the number density. The closure equation is expressed by [6,9-12]

$$
\begin{aligned}
& c_{\alpha \beta}(12)=\int_{r}^{\infty}\left[h_{\alpha \beta}(12) \partial\left\{w_{\alpha \beta}(12)-b_{\alpha \beta}(12)\right\} / \partial r\right] d r-u_{\alpha \beta}(12) /\left(k_{\mathrm{B}} T\right)+b_{\alpha \beta}(12), \\
& w_{\alpha \beta}(12)=-\eta_{\alpha \beta}(12)+u_{\alpha \beta}(12) /\left(k_{\mathrm{B}} T\right),
\end{aligned}
$$

where $u$ is the pair potential, $b$ is the bridge function, and $r$ is the distance between the centers of two particles. The hypernetted-chain (HNC) approximation is employed $(b=0)$ in the present study.

Since the solutes are present at infinite dilution $\left(\rho_{\mathrm{U}}=0\right)$, the calculation process can be split into two steps: 
(i) Solve Eqs. (1) and (2) for water. Calculate the correlation functions $X_{\mathrm{SS}}(X=h, c)$.

(ii) Solve Eqs. (1) and (2) for water in which a solute is immersed using the correlation functions obtained in step (i) as input data. Calculate the correlation functions $X_{\mathrm{US}}$ $(X=h, c)$.

Equations (1) and (2), which comprise multi-variable functions and multiple integrations, are not numerically tractable. Therefore, they need to be reduced mathematically. The pair potentials and correlation functions are expanded in a basis set of rotational invariants, and the basic equations are reformulated in terms of the projections $X_{\alpha \beta}{ }^{m n l}{ }_{\mu v}(r)$ occurring in the rotational-invariant expansion of $X_{\alpha \beta}(12)$ : The angular components are analytically treated using the orthogonality of Wigner's generalized spherical harmonics [6,9-12]. The expansion considered for $m, n \leq n_{\max }=4$ gives sufficiently accurate results. The basic equations are then numerically solved using the robust, highly efficient hybrid algorithm developed by Kinoshita et al. [13]. In the numerical treatment, a sufficiently long range $r_{\mathrm{L}}$ is divided into $N$ grid points $\left(r_{i}=i \delta r, i=0,1, \ldots, N-1 ; \delta r=r_{\mathrm{L}} / N\right)$ and all of the projections are represented by their values on these points. The grid width and the number of grid points are set at $\delta r=0.01 d_{\text {s }}$ and $N=4096$, respectively.

\subsection{Calculation of hydration free energy and its entropic and energetic components}

Since the HNC approximation is employed, the HFE (i.e., excess chemical potential) $\mu$ can be calculated using the Morita-Hiroike formula [14] written as

$$
\begin{aligned}
\mu /\left(k_{\mathrm{B}} T\right) & =\left\{\rho_{\mathrm{S}} /\left(8 \pi^{2}\right)\right\} \iiint \int 4 \pi\left[\left\{h_{\mathrm{US}}(r, \theta, \phi, \chi)\right\}^{2} / 2-h_{\mathrm{US}}(r, \theta, \phi, \chi) C_{\mathrm{US}}(r, \theta, \phi, \chi) / 2\right. \\
& \left.-c_{\mathrm{US}}(r, \theta, \phi, \chi)\right] r^{2} \sin \theta d r d \theta d \phi d \chi
\end{aligned}
$$

where the integration range is $[0, \infty]$ for $r,[0, \pi]$ for $\theta$, and $[0,2 \pi]$ for $\phi$ and $\chi$. We have shown the following: The HFE of a hard-sphere solute with diameter $d_{\mathrm{U}}=0.28 \mathrm{~nm}$ is calculated to be $3.52 \mathrm{kcal} / \mathrm{mol}$ [11] that is in very good agreement with the values from Monte Carlo simulations for more popular water models: $3.52 \mathrm{kcal} / \mathrm{mol}$ for TIP4P [15] and $3.65 \mathrm{kcal} / \mathrm{mol}$ for SPC/E [16].

It should be noted that $\mu$ is independent of the solute insertion condition, isobaric or isochoric, but the hydration entropy $S$ and energy $U$ are not [6,7]. We consider isochoric condition and $S$ is calculated from

$S=-(\partial \mu / \partial T)_{V}$

where the subscript " $V$ " denotes the isochoric condition and the temperature derivatives are numerically evaluated from 
$(\partial \mu / \partial T)_{V}=\{\mu(T+\delta T)-\mu(T-\delta T)\} /(2 \delta T), \delta T=5 \mathrm{~K}$.

The hydration energy $U$ is then obtained from

$U=\mu+T S$.

Each of the three quantities $(\mu, S$, and $U$ ) comprises the translational and rotational components. However, as shown in our earlier works [1,17], the translational component predominates over the rotational one.

The reduced density profile of water, $g(r)=h_{\mathrm{US}}{ }^{000}{ }_{00}(r)+1$, is also important for small separations $(g(r)$ is often referred to as the solute-water pair correlation function). We look at its contact value, $g\left(d_{\mathrm{US}}\right)\left(d_{\mathrm{US}}=\left(d_{\mathrm{U}}+d_{\mathrm{S}}\right) / 2\right)$.

\section{Results and Discussion}

\subsection{Change in hydrophobic hydration caused by raising the pressure}

Table 1 gives $-T S, U, \mu$, and $g\left(d_{\mathrm{US}}\right)$ calculated for the hydrophobic solute at the low and high pressures. "Difference" represents "value at the high pressure" minus "value at the low pressure". $-T S$ and $U$ are, respectively, the entropic and energetic components of $\mu$. The solute insertion causes an entropic loss $(S<0)$. For a solvent whose molecules interact through attractive potential like water, the insertion of a solute generates an excluded volume and causes the solvent energy to decrease $(U<0)$. Since the direct solute-water interaction does not contribute energetically for our solute model, the energy decrease is due to the structural changes induced in the bulk water. (At the temperature that is low enough to induce cold denaturation of a protein, the structured water near the solute also contributes to the energy decrease. The denaturation leads to energy decreases for the bulk water and for the structured water near the protein due to large increases in the excluded volume and the water-accessible surface area, respectively.) Our finding for the solute-size dependence is the following: As $d_{U}$ becomes larger, both $-T S$ and $|U|$ increase but the increase in the former is substantially larger with the result of a large increase in $\mu$.

Upon raising the pressure, $-T S$ changes in the direction that $\mu$ increases whereas the opposite is true for the change in $U$. However, the change in $-T S$ is $~ 5.1$ times larger than that in $U$. As a consequence, $\mu$ becomes substantially higher. This tendency becomes stronger with increasing $d_{\mathrm{U}}$ (the results are not shown). It is observed that $g\left(d_{\mathrm{US}}\right)$ increases as the pressure becomes higher. As explained in the second paragraph of Introduction, the water crowding in the bulk is serious at high pressures and reducing it is crucial with the result that many water molecules are forced to contact the solute. 


\subsection{Change in hydrophilic hydration caused by raising the pressure}

Table 2 gives $-T S, U, \mu$, and $g\left(d_{\mathrm{US}}\right)$ calculated for the hydrophilic solute at the low and high pressures. The behavior of $-T S$ for the hydrophilic solute is quantitatively similar to that for the hydrophobic solute. However, due to the solute-water electrostatic (charge-dipole and charge-quadrupole) interaction which is attractive, $U$ becomes much more negative, making $\mu=U-T S$ negative. Water molecules are attracted to the solute, and $g\left(d_{\text {US }}\right)$ in the hydrophilic hydration is larger than that in the hydrophobic one.

Upon raising the pressure, $-T S$ changes in the direction that $\mu$ increases whereas the opposite is true for the change in $U$. However, the change in $-T S$ is $\sim 2.8$ times larger than that in $U$. As a consequence, $\mu$ becomes substantially higher. This tendency becomes stronger with increasing $d_{\mathrm{U}}$.

\subsection{Change in hydrophobic hydration caused by lowering the temperature}

Table 3 gives $-T S, U, \mu$, and $g\left(d_{\mathrm{US}}\right)$ calculated for the hydrophobic solute at the three temperatures. "Difference” represents "value at $258 \mathrm{~K}$ ” minus "value at $298 \mathrm{~K}$ ”.

Upon lowering $T$, both -TS and $U$ change in the direction that $\mu$ decreases and the change in the former is $\sim 2.5$ times larger. As $T$ becomes lower, $\mu$ decreases to a considerable extent and even the measure of solute hydrophobicity, $\mu /\left(k_{\mathrm{B}} T\right)$, decreases. This tendency becomes stronger with increasing $d_{\mathrm{U}}$. It is observed that $g\left(d_{\mathrm{US}}\right)$ decreases as $T$ becomes lower, which implies that water dislikes the solute more from the viewpoint of the solute-water pair correlation. The decrease in $\mu /\left(k_{\mathrm{B}} T\right)$ is attributable to the reduction of the water crowding which can be understood only on the basis of the solute-water many-body correlation.

\subsection{Change in hydrophilic hydration caused by lowering the temperature}

Table 4 gives $-T S, U, \mu$, and $g\left(d_{\mathrm{US}}\right)$ calculated for the hydrophilic solute at the four temperatures. "Difference" represents "value at $258 \mathrm{~K}$ ” minus "value at $298 \mathrm{~K}$ ". The behavior of -TS for the hydrophilic solute is quantitatively similar to that for the hydrophobic solute. However, due to the solute-water electrostatic attractive interaction, $U$ becomes much more negative, making $\mu=U-T S$ negative. (The decrease in the energy of the bulk water upon solute insertion is the same for hydrophobic and hydrophilic hydrations.)

Upon lowering $T,-T S$ changes in the direction that $\mu$ decreases whereas the opposite is true for the change in $U$. As $T$ becomes lower, above $273 \mathrm{~K}$ the former effect dominates and a decrease in $\mu$ is observed. Below $273 \mathrm{~K}$, however, the inversion occurs: The latter effect dominates and $\mu$ exhibits an increase. This temperature 
dependence holds for the measure of solute hydrophilicity, $\mu /\left(k_{\mathrm{B}} T\right)$, as well. At $258 \mathrm{~K}$, the solute is considerably less hydrophilic. This behavior is also reflected on $g\left(d_{\mathrm{US}}\right)$ which first increases as $T$ becomes lower but decreases below $273 \mathrm{~K}$. We have examined the three more combinations of the values of $\left(q_{\mathrm{U}} / \mathrm{e}, d_{\mathrm{U}} / \mathrm{d}_{\mathrm{S}}\right):(0.5,1),(0.7,1)$, and $(0.5,0.8)$. The characteristics described above are robustly observed.

With decreasing $T$, the effective solute-water electrostatic attractive interaction (i.e., the interaction scaled by $k_{\mathrm{B}} T$ ) becomes stronger. If this effect dominated, $U$ would decrease because the strengthened effective solute-water electrostatic attractive interaction brings a decrease in energy (this is referred to as "energy decrease"). However, Table 4 shows that the opposite is true. As $T$ is more lowered, the water structure near the solute is more perturbed due to the strengthened effective solute-water electrostatic attractive interaction, causing the break of hydrogen bonds and an increase in energy (this is referred to as "energy increase”). Since $U$ increases as $T$ becomes lower as observed in Table 4, the energy increase is larger than the energy decrease: Above $273 \mathrm{~K}$ the difference becomes larger only slightly as $T$ decreases, but below $273 \mathrm{~K}$ it becomes larger rather markedly. This is because below $273 \mathrm{~K}$ the water-water hydrogen bonding is remarkably enhanced with the result that the energy increase arising from the break of hydrogen bonds is substantially enlarged. When $T$ is lowered from $273 \mathrm{~K}$, the solute becomes less hydrophilic in the sense that $\mu /\left(k_{\mathrm{B}} T\right)$ shifts in the positive direction (see Table 4 ). Since most of the proteins undergo cold denaturation below $273 \mathrm{~K}$ [18], the significant result is that a hydrophilic group in a protein becomes less hydrophilic at the low temperatures relevant to cold denaturation.

\subsection{Summary}

The pressure and temperature dependence of the entropic component of the HFE for the hydrophobic solute is similar to that for the hydrophilic solute. When the solute diameter is the same, the entropic component behaves similarly even in a quantitative sense regardless of the solute hydrophobicity or hydrophilicity. This is not the case for the energetic component. Except for hydrophilic hydration below $273 \mathrm{~K}$, the pressure, temperature, and solute-size dependence of the entropic component is stronger than that of the energetic component. In particular, the pressure dependence of the HFE is governed by that of the entropic component.

\subsection{Effects of solute-water van der Waals potential}

We briefly discuss the effects of solute-water van der Waals attractive potential (the results are not shown). When it is newly incorporated in the calculation, $U$ and $\mu$ become lower but -TS remains almost unchanged. This is true for both of hydrophobic and hydrophilic solutes. Moreover, the magnitudes of the changes of $U$ and $\mu$ are much 
smaller than those in the case where solute-water electrostatic (attractive) potential is newly incorporated. The important results summarized in 3.5 are not altered at all by the incorporation of the van der Waals potential. Its effects are insensitive to the pressure or temperature change.

\section{Validity of our previous studies on pressure and cold denaturating of proteins}

\subsection{Pressure denaturation}

Our physical picture of pressure denaturation of a protein was developed on the basis of theoretical analyses considering only the entropic component of the HFE. Moreover, a protein comprising only hydrophobic groups was treated [1]. Upon raising the pressure, the entropic component exhibits a large change, whereas the change in the energetic component remains much smaller. Since the entropic component behaves similarly for both of hydrophobic and hydrophilic solutes, the hydrophilic groups can be replaced by hydrophobic groups. We can conclude that a theoretical approach considering only hydrophobic groups and the entropic component of the HFE is acceptable in elucidating the physical essence of pressure denaturation. Since solute-water van der Waals potential does not influence the solute hydrophobicity, the neglect of protein-water van der Waals potential in our earlier work [1] can be justified.

\subsection{Cold denaturation}

In the development of the physical picture of cold denaturation of a protein, both of the entropic and energetic components were taken into account, but a protein comprising only hydrophobic groups was treated [2-4]. The present result suggests that a hydrophilic group becomes less hydrophilic at low temperatures. Therefore, the factor promoting the exposure of hydrophilic groups to water becomes less powerful at low temperatures. The presence of hydrophilic groups is not relevant to cold denaturation. Since solute-water van der Waals potential does not influence the solute hydrophobicity and hydrophilicity, the neglect of protein-water van der Waals potential in our earlier works [2-4] can be justified.

\subsection{Further discussion}

When the change in the "intramolecular energy plus hydration energy" upon protein folding, $\Delta E$, is analyzed, protein-water electrostatic and van der Waals potentials are to be taken into account. (If the protein possesses only a rather small total charge, the net effect of van der Waals potential could be more important than that 
of electrostatic potential $[19,20]$.) $\Delta E$ is largely influenced by electrostatic and van der Waals potentials, whereas the change in the hydration entropy is not. To the pressure or temperature change, $\Delta E$ is much less sensitive than the change in the hydration entropy. In accordance with the discussion in the last paragraph of the Introduction, both of electrostatic and van der Waals potentials can be neglected in studies on pressure and cold denaturating of a protein. (We emphasize that the justification of the neglect is not general: It is limited to studies on pressure and cold denaturating.)

\section{Concluding Remarks}

A self-assembly process such as protein folding and receptor-ligand binding is accompanied by an energy decrease due to the gain of intramolecular and intermolecular interactions for biomolecules (factor 1). In many cases, however, factor 1 is almost cancelled out by the energy increase originating from the energetic dehydration effect (factor 2) [8]. Factor 2 represents the loss of water-biomolecule interactions and the energy change due to structural reorganization of water. It is often that factor 2 is larger than factor 1 and the net energy change is positive $[8,17]$. The process is driven by the water-entropy gain surpassing the loss of conformational entropy of biomolecules and the sum of factors 1 and $2[8,17,21]$.

The structures formed by biological self-assembly are collapsed by the application of high pressures. Typical examples are the dissociation of amyloid fibrils [22], dissociation of F-actin into G-actin monomers [23], and pressure denaturation of a protein. The power of the formation is weakened at low temperatures. Typical examples are the weakening of the binding power of myosin to F-actin [24] and cold denaturation of a protein. Such phenomena are not limited to the biological system. For example, for nonionic surfactant molecules, the critical micelle concentration increases and the average micelle size decreases at low temperatures [25]. These experimental results suggest that the self-assembly processes share the same feature and a certain physical factor universally plays a dominant role as the driving force. We have shown that the factor is the solute-water many-body correlation component of the entropic excluded-volume effect (i.e., the effect of the self-assembly on the water crowding) and this is the true physical origin of hydrophobicity [21]. The self-assembly processes are driven by the hydrophobic effect. We emphasize that even for a hydrophilic solute the hydrophobic effect certainly works (note that the entropic component is always positive) and competes with the other factors. 


\section{Acknowledgment}

This work was supported by JSPS (Japan Society for the Promotion of Science) Grants-in-Aid for Scientific Research (B) (No. 25291035: M. Kinoshita) and by Grant-in-Aid for JSPS fellows (H. Oshima).

\section{References}

[1] Y. Harano, T. Yoshidome, M. Kinoshita, J. Chem. Phys. 129 (2008) 145103.

[2] T. Yoshidome, M. Kinoshita, Phys. Rev. E 79 (2009) 030905(R).

[3] H. Oshima, T. Yoshidome, K. Amano, M. Kinoshita, J. Chem. Phys. 131 (2009) 205102(1-11).

[4] T. Yoshidome, M. Kinoshita, Phys. Chem. Chem. Phys. 14 (2012) 14554.

[5] H. Oshima and M. Kinoshita, J. Chem. Phys. 138 (2013) 245101.

[6] N. M. Cann, G. N. Patey, J. Chem. Phys. 106 (1997) 8165.

[7] M. Kinoshita, Y. Harano, R. Akiyama, J. Chem. Phys. 125 (2006) 244504.

[8] T. Hayashi, H. Oshima, T. Mashima, T. Nagata, M. Katahira, M. Kinoshita, Nucleic Acids Res. 42 (2014) 6861.

[9] P. G. Kusalik, G. N. Patey, J. Chem. Phys. 88 (1988) 7715.

[10] P. G. Kusalik, G. N. Patey, Mol. Phys. 65 (1988) 1105.

[11] M. Kinoshita, J. Chem. Phys. 128 (2008) 024507.

[12] M. Kinoshita, T. Yoshidome, J. Chem. Phys.130 (2009) 144705.

[13] M. Kinoshita, D. R. Bérard, J. Comput. Phys. 124 (1996) 230.

[14] T. Morita, K. Hiroike, Prog. Theor. Phys. 25 (1961) 537.

[15] M. Ikeguchi, S. Shimizu, S. Nakamura, K. Shimizu, J. Phys. Chem. B 102 (1998) 5891.

[16] N. Matubayasi, M. Nakahara, J. Chem. Phys. 112 (2000) 8089.

[17] T. Yoshidome, M. Kinoshita, S. Hirota, N. Baden, M. Terazima, J. Chem. Phys. 128 (2008) 225104.

[18] A. Pastore, S. R. Martin, A. Politou, K. C. Kondapalli, T. Stemmler, P. A. Temussi, J. Am. Chem. Soc. 129 (2007) 5374.

[19] T. Imai, Y. Harano, M. Kinoshita, A. Kovalenko, and F. Hirata, J. Chem. Phys. 126 (2007) 225102.

[20] T. Hayashi, H. Oshima, T. Mashima, T. Nagata, M. Katahira, and M. Kinoshita, Nucleic Acids Res. 42 (2014) 6861.

[21] M. Kinoshita, Biophys. Rev. 5 (2013) 283.

[22] D. Foguel, M. C. Suarez, A. D. Ferrão-Gonzales, T. C. R. Porto, L. Palmieri, C. M. Einsiedler, L. R. Andrade, H. A. Lashuel, P. T. Lansbury, J. W. Kelly, J. L. Silva, Proc. Natl. Acad. Sci. USA 100 (2003) 9831.

[23] T. Ikkai, T. Ooi, Biochemistry 5 (1966) 1551. 
[24] T. Katoh, F. Morita, J. Biochem. (Tokyo) 120 (1996) 189.

[25] D. Myers, Surfaces, Interfaces, and Colloids: Principles and Applications, 2nd ed., Wiley-VCH, Berlin, 1999. 
Table 1. Change in hydrophobic hydration properties caused by raising the pressure.

\begin{tabular}{ccccc}
$\rho \mathrm{s}_{\mathrm{s}}{ }^{3}$ & $-T S(\mathrm{kcal} / \mathrm{mol})$ & $U(\mathrm{kcal} / \mathrm{mol})$ & $\mu(\mathrm{kcal} / \mathrm{mol})$ & $g\left(d_{\mathrm{Us}}\right)$ \\
\hline 0.7317 & 24.88 & -13.21 & 11.67 & 1.812 \\
0.8500 & 40.39 & -16.27 & 24.12 & 3.706 \\
Difference & 15.51 & -3.06 & 12.45 & 1.894
\end{tabular}

Table 2. Change in hydrophilic hydration properties caused by raising the pressure.

\begin{tabular}{|c|c|c|c|c|}
\hline$\rho_{\mathrm{s}} d_{\mathrm{s}}{ }^{3}$ & $-T S(\mathrm{kcal} / \mathrm{mol})$ & $U(\mathrm{kcal} / \mathrm{mol})$ & $\mu(\mathrm{kcal} / \mathrm{mol})$ & $g\left(d_{\mathrm{US}}\right)$ \\
\hline 0.7317 & 24.20 & -62.31 & -38.11 & 5.780 \\
\hline 0.8500 & 41.10 & -68.42 & -27.32 & 7.207 \\
\hline Difference & 16.90 & -6.11 & 10.79 & 1.427 \\
\hline
\end{tabular}

Table 3. Change in hydrophobic hydration properties caused by lowering the temperature.

\begin{tabular}{ccccc}
$T(\mathrm{~K})$ & $-T S(\mathrm{kcal} / \mathrm{mol})$ & $U(\mathrm{kcal} / \mathrm{mol})$ & $\mu(\mathrm{kcal} / \mathrm{mol})$ & $g\left(d_{\mathrm{Us}}\right)$ \\
\hline 298 & 24.88 & -13.21 & 11.67 & 1.812 \\
273 & 23.02 & -13.29 & 9.73 & 1.565 \\
258 & 22.46 & -14.19 & 8.27 & 1.338 \\
Difference & -2.42 & -0.98 & -3.40 & -0.474
\end{tabular}


Table 4. Change in hydrophilic hydration properties caused by lowering the temperature.

\begin{tabular}{ccccc}
$T(\mathrm{~K})$ & $-T S(\mathrm{kcal} / \mathrm{mol})$ & $U(\mathrm{kcal} / \mathrm{mol})$ & $\mu(\mathrm{kcal} / \mathrm{mol})$ & $g\left(d_{\mathrm{Us}}\right)$ \\
\hline 323 & 26.61 & -63.09 & -36.48 & 5.630 \\
298 & 24.20 & -62.31 & -38.11 & 5.780 \\
273 & 21.31 & -61.30 & -39.99 & 5.916 \\
258 & 20.20 & -54.74 & -34.54 & 5.345 \\
Difference & -4.00 & 7.57 & 3.57 & -0.435
\end{tabular}

\section{Figure Captions}

Fig. 1. Contact of a water molecule with the solute.

Fig. 2. Characteristics of pressure denaturation of a protein. "Denatured Structure" is a representative structure of the denatured state.

Fig. 3. Characteristics of cold denaturation of a protein. "Denatured Structure" is a representative structure of the denatured state. 


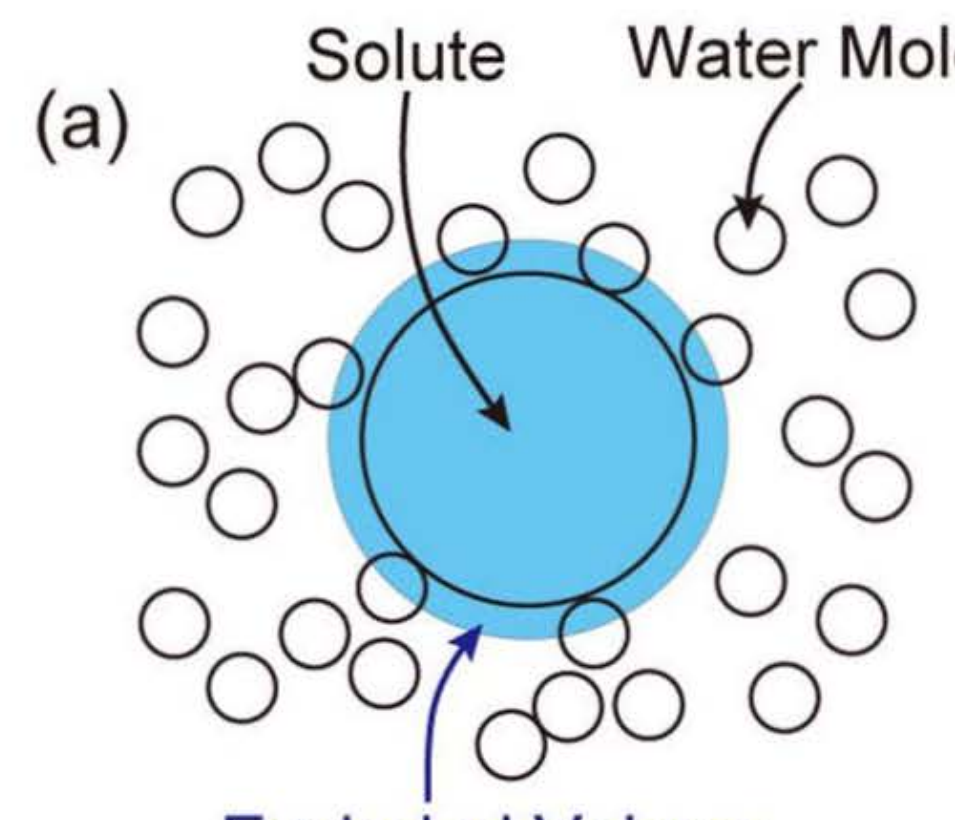

Excluded Volume Generated by a Solute (b)

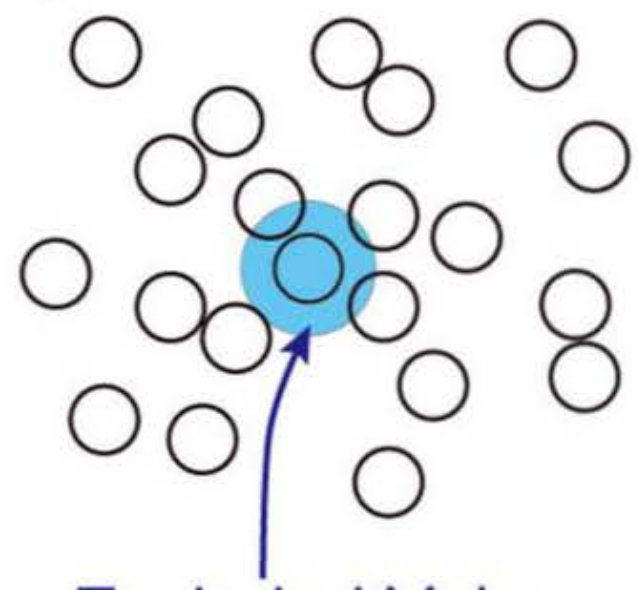

Excluded Volume Generated by a Water Molecule

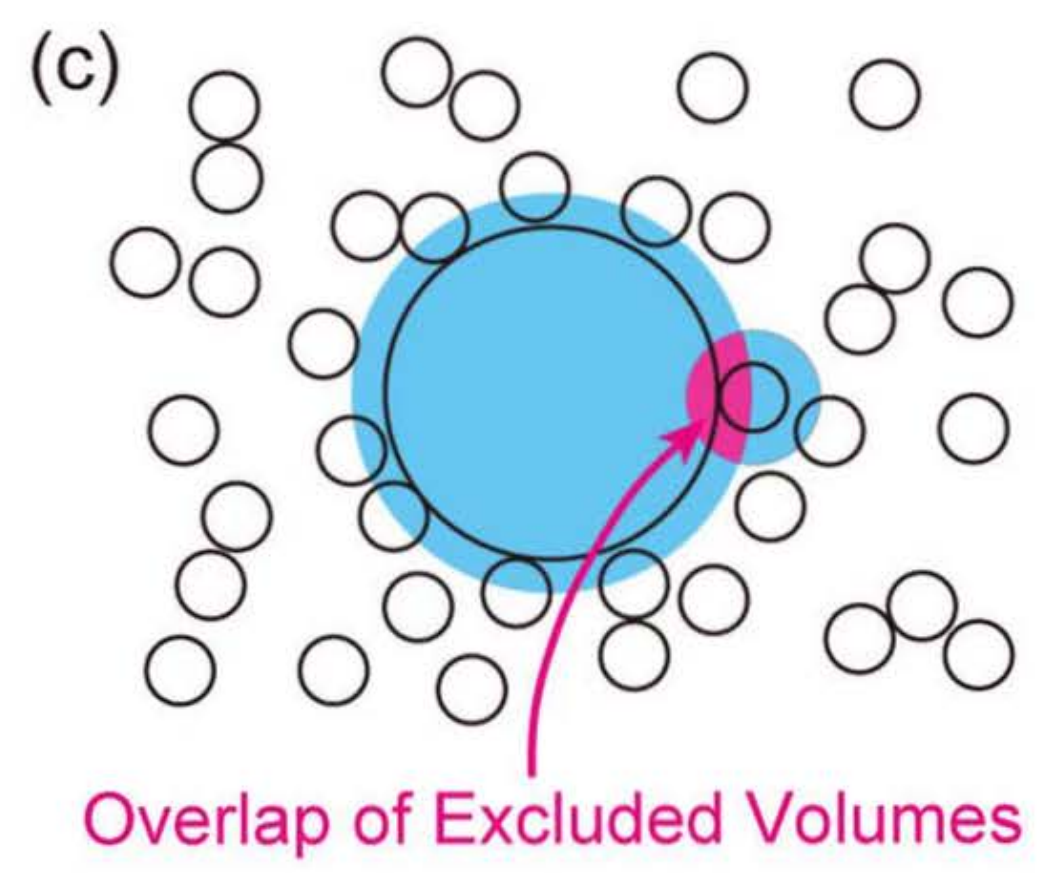

Fig. 1. 


\section{Native Structure}

\section{Denatured Structure}

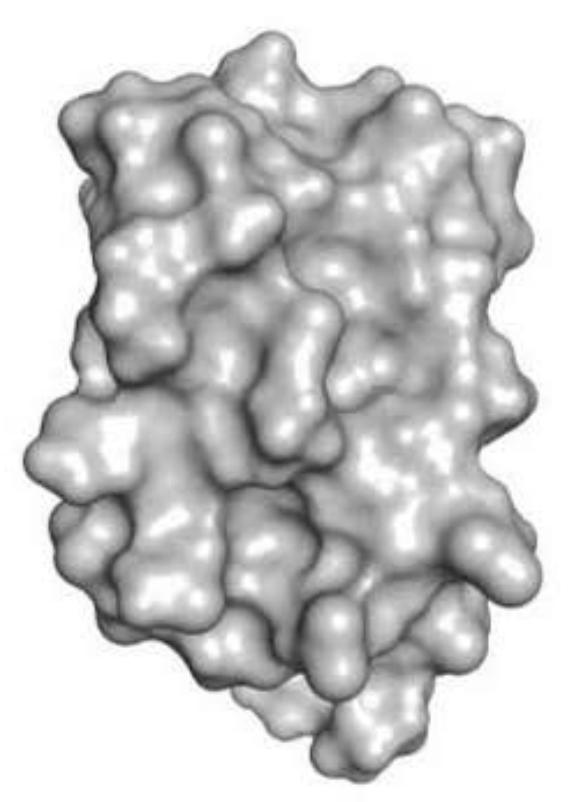

Excluded Volume

Water-Accessible Surface Area Water Entropy (Low Pressure) Water Entropy (High Pressure)
Slight increase in excluded volume.

Decrease in partial molar volume.

Water penetration: Swelling.

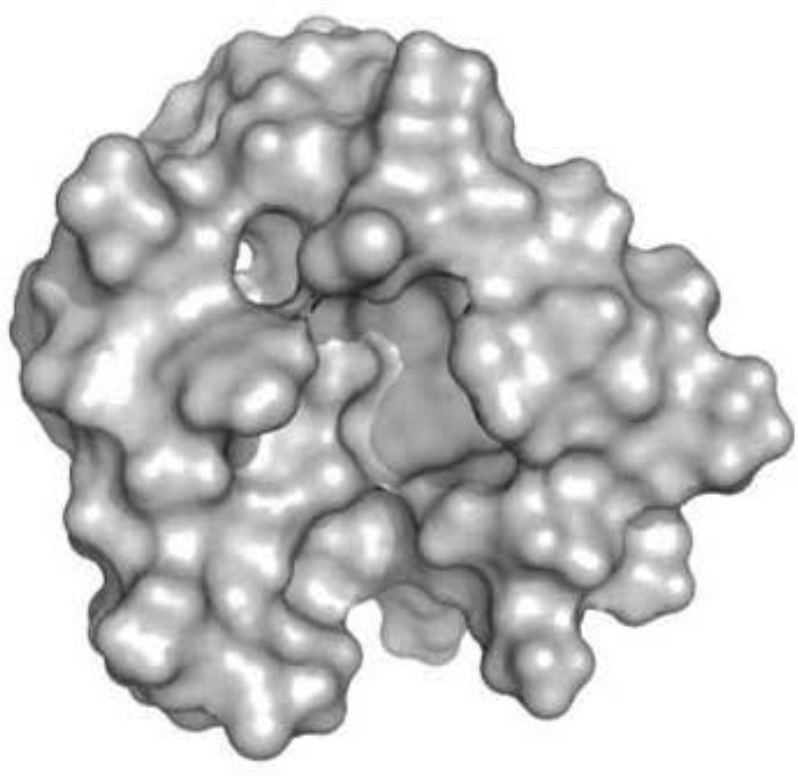
$<\quad$ Excluded Volume < Water-Accessible Surface Area $>\quad$ Water Entropy (Low Pressure) $<\quad$ Water Entropy (High Pressure) 


\section{Native Structure}

\section{Denatured Structure}
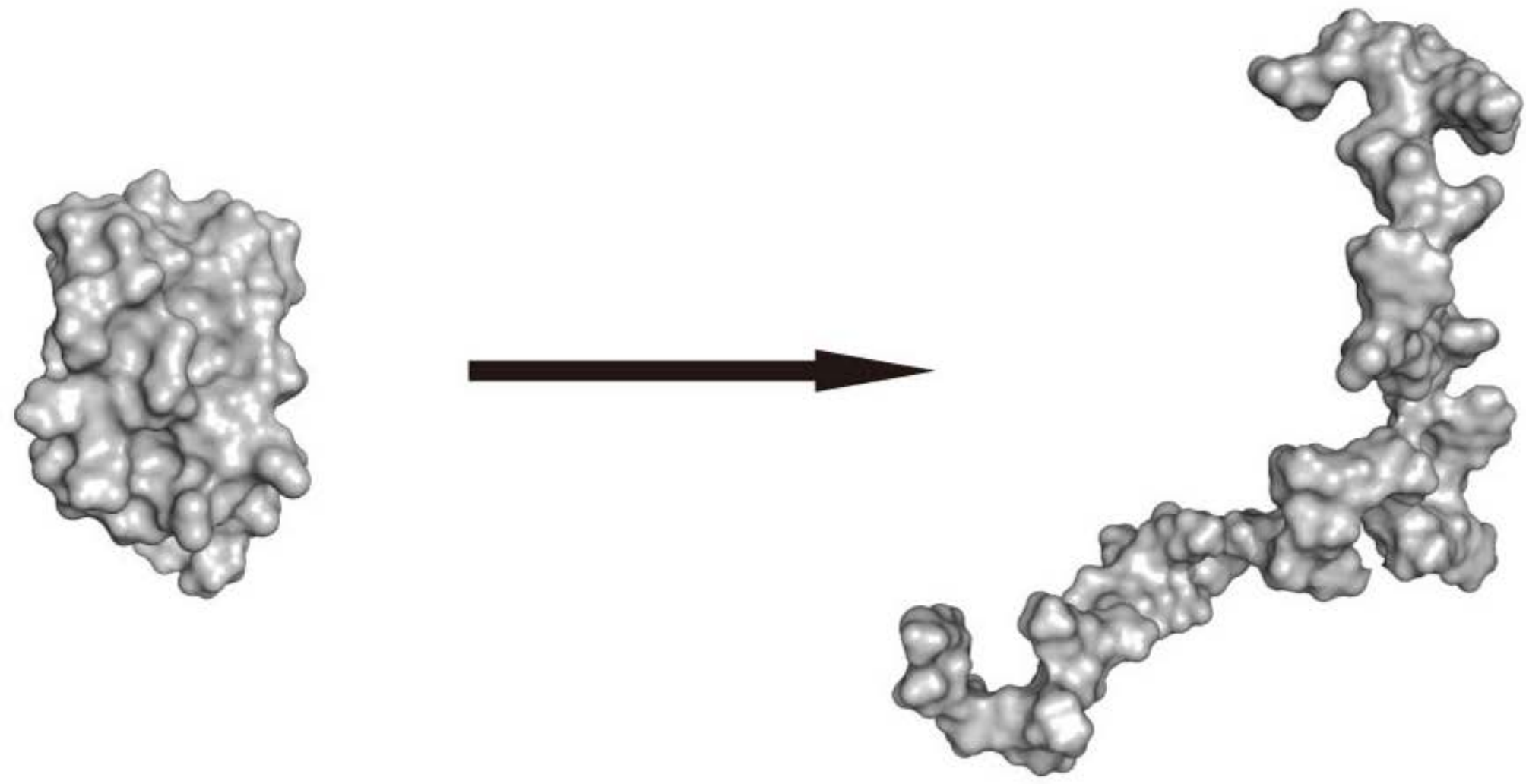

Excluded Volume Water-Accessible Surface Area Water Entropy

System Entropy

System Energy

$\begin{array}{cr}\ll< & \text { Excluded Volume } \\ \ll & \text { Water-Accessible Surface } \\ >> & \text { Water Entropy } \\ > & \text { System Entropy } \\ > & \text { System Energy }\end{array}$

Fig. 3. 